\title{
Structure-Activity Relationship (SAR) and in vitro Predictions of Mutagenic and Carcinogenic Activities of Ixodicidal Ethyl- Carbamates
}

\author{
María G. Prado-Ochoa, ${ }^{1}$ Maribel Strassburger-Madrigal, ${ }^{2}$ Rafael Camacho-Carranza, ${ }^{3}$ \\ Jesús J. Espinosa-Aguirre, ${ }^{3}$ Ana M. Velázquez-Sánchez, ${ }^{4}$ Victor H. Vázquez-Valadez, ${ }^{4}$ \\ Enrique Angeles, ${ }^{4}$ Fernando Alba-Hurtado, ${ }^{1}$ and Marco A. Muñoz-Guzmán $\left(\mathbb{D}{ }^{1}\right.$ \\ ${ }^{1}$ Departamento de Ciencias Biológicas, Facultad de Estudios Superiores Cuautitlán, \\ Universidad Nacional Autónoma de México, Mexico \\ ${ }^{2}$ Programa de Maestría en Ciencias de la Producción y de la Salud Animal, Universidad Nacional Autónoma de México, Mexico \\ ${ }^{3}$ Departamento de Medicina Genómica y Toxicología Ambiental, Instituto de Investigaciones Biomédicas, \\ Universidad Nacional Autónoma de México, Mexico \\ ${ }^{4}$ Laboratorio de Química Medicinal, Departamento de Ciencias Químicas, Facultad de Estudios Superiores Cuautitlán, \\ Universidad Nacional Autónoma de México, Mexico
}

Correspondence should be addressed to Marco A. Muñoz-Guzmán; mmunoz74@hotmail.com

Received 29 July 2020; Revised 15 October 2020; Accepted 5 November 2020; Published 21 November 2020

Academic Editor: Peyman Björklund

Copyright ( 2020 María G. Prado-Ochoa et al. This is an open access article distributed under the Creative Commons Attribution License, which permits unrestricted use, distribution, and reproduction in any medium, provided the original work is properly cited.

\begin{abstract}
Ethyl-4-bromophenyl-carbamate (LQM 919) and Ethyl-4-chlorophenyl-carbamate (LQM 996) are compounds that inhibit egglaying and hatching of tick larvae that are resistant to conventional ixodicides. The structure-activity relationship (SAR) to get the endpoint predictions of mutagenicity and carcinogenicity of the LQM 919 and LQM 996 was performed and the absence of mutagenicity was confirmed by Ames test. SAR analysis show no structural alerts indicating the ability of ethyl-carbamates to bind biomolecules or estrogen receptors. Endpoint of mutagenicity with and without metabolic activation showed that the ethylcarbamates were negative $(\mathrm{p}<0.05)$ for mutagenicity induction in strains TA97, TA98, TA102, TA1535, TA1537 and TA1538 of Salmonella typhimurium. Pre-incubation with different ethyl-carbamate concentrations did not increase the number of spontaneously reverting colonies; moreover, the compounds did not induce a concentration-dependent increase in the number of reverting colonies in any of the strains used. This confirmed the absence of mutagenic activity in this test system. Exogenous metabolic activation did not modify these observations; suggesting that no metabolites with mutagenic activity were present. The endpoint of carcinogenicity in rats were negative for LQM $919(\mathrm{p}<0.05$,) and LQM $996(\mathrm{p}<0.001)$. The results of the present study strongly suggest that ethyl-carbamates do not represent a risk for cancer in mammals.
\end{abstract}

\section{Introduction}

Currently, regulation of chemical substances that are used daily as pesticides (i.e. ixodicides) is of great relevance globally. The European Chemicals Agency (ECHA), the Organization for Economic Cooperation and Development (OECD), and the Environmental Protection Agency (EPA), are the most important organizations responsible for setting the guidelines for regulation of chemical substances.
These organizations generally accept in silico methods based on computational modeling of chemical structure and biological activity of a compound to predict its toxicity without the use of biological tests. This allows reducing costs, the number of experimental animals, risks in handling hazardous chemicals, and the time used to perform in vitro or in vivo tests [1].

Rhipicephalus microplus, considered the most important parasitic tick on cattle in tropical and subtropical areas 
worldwide, represents a great threat to the livestock industry due to the diseases it transmits as a vector and the direct economic losses it causes [2]. Excessive use of chemical substances for the control of ticks in cattle (ixodicides) has resulted in development of tick strains that are resistant to the majority of commercial ixodicides in several regions of the world [3]. This has generated the need to develop new molecules to which no tick strains are currently resistant. We have shown that ethyl-4-bromophenyl-carbamate (LQM 919) and ethyl-4-chlorphenyl-carbamate (LQM 996) inhibit egg-laying and hatching of Rhipicephalus microplus larvae and this inhibition was observed both in strains that are resistant to other ixodicides and in those that are susceptible to them $[4,5]$. These carbamates alter the reproductive organs, vitellogenesis, and viability of ovarian cells in $R$. microplus and cause harm that is suggestive of apoptosis [6]. These effects were found to be independent of acetylcholinesterase inhibition [7].

The toxicity of LQM 919 and LQM 996 must be evaluated before these compounds are proposed for the safe control of ticks. Following the criteria of the OECD, these carbamates are found to have low acute and subchronic toxicity in rats $[8,9]$. Nevertheless, these compounds produce an increase in micronucleated reticulocytes in the peripheral blood of rats as well as alterations in the cell cycle of human lymphocytes suggesting that these compounds cause DNA damage [10]. Other carbamates such as vinyl-carbamate and ethyl-carbamate (urethane) are carcinogenic and produce tumors in several organs of rodents $[11,12]$. This suggests a potential risk for the application of carbamates in animals used for human food and to the persons who apply these products. Therefore, this study aimed to evaluate the mutagenic and carcinogenic potential of the ethylcarbamates LQM 919 and LQM 996 in silico, as well as to corroborate their mutagenic capacity using the Ames test.

\section{Materials and Methods}

2.1. Carbamates. The ethyl-carbamates used in this study were designed, synthesized, and patented at the National Autonomous University of Mexico using a benzimidazole molecule as the structural base, as described by Angeles et al. [13]. The chemical structures, nomenclature, molecular weights, simplified molecular input line entry system (SMILES), octanol/water partition coefficient (Kow) and identification codes of ethyl-carbamates are shown in Table 1.

2.2. In silico Studies. Structure-activity relationship (SAR) for endpoint predictions of mutagenicity and carcinogenicity of the ethyl-carbamates LQM 919 and LQM 996 was performed by Read-across-analysis using the QSAR Toolbox software according to workflow of the hazard assessment process: Input; Profiling; Endpoint data collection; category definition; data gap filling, and finally, reporting [14]. The partition coefficients ( $\log \mathrm{Kw}$ ) of each carbamate were used as descriptors. A conformational study of LQM 919 and LQM 996 was conducted; the molecules were modeled using the Toolbox modeler tool and were double checked by compar- ing each SMILES code. Profiling was carried out using the OECD HPV chemical categories, substance type, and USEPA New Chemical Categories profiling methods $[15,16]$.

The carbamate structures were optimized by PM3 (Molecular Orbital Model) and were categorized using the DICE (Dynamic Integrated model of Climate and the Economy) model to obtain the DICE coefficient $[17,18]$. Molecular feature parameters (atom centered, fragments and cycles) and atomic characteristics (count $\mathrm{H}$ attach, hybridization and cyclic) were used to obtain a set of chemical analogues with greater than $50 \%$ similarity to the carbamates LQM 919 and LQM 996. The endpoint predictions of mutagenicity on strains TA97, TA98, TA102, TA1535, TA1537, and TA1538 of Salmonella typhimurium with and without simulation of S9 metabolic activation, and endpoint predictions of carcinogenicity on rats by the oral route of ethyl-carbamates were determined. The endpoint value predictions were based on five neighbors' values. Prediction confidence was established and categorized as moderate $(\mathrm{p}<0.05)$, strong $(\mathrm{p}<$ $0.01)$, or very strong $(\mathrm{p}<0.001)$.

2.3. Ames' in vitro Assay. S. typhimurium strains TA98 (his D3052, rfa National Autonomous University of Mexico, uvrB, bio-, pKM101[ApR]), TA100 (his G46, rfa, uvrB, bio, pKM101[ApR]), TA1535 (his G46, rfa, uvrB, bio-), TA1537 (his C3076, rfa, uvrB, bio-), and TA102 (His G428, rfa, bio-, pKM101[ApR], pAQ1[TtR]) were used; these were maintained at the Mutagens and Carcinogens Laboratory of the Biomedical Research Institute, National Autonomous University of Mexico. Before the assays were carried out, single colonies were isolated from each strain, and their genotypic characteristics were confirmed using the procedure described by Maron and Ames [19]. The toxicity of varying concentrations of the studied compounds was assessed by microscopic observation of bacterial growth in the selected dishes. Homogeneous distribution of bacteria was considered indicative of no toxicity, whereas loss of homogeneity and the dose-dependent decrease in reverting colonies was considered indicative of toxicity.

Extract S9 from the livers of Sprague-Dawley male rats, induced with phenobarbital and B-naphthoflavone, was prepared and stored $\left(-80^{\circ} \mathrm{C}\right)$ according to the modified method described by Escobar-García et al. [20].

One colony from each strain grown in NB solid medium was transferred to $10 \mathrm{~mL}$ of NB-Oxoid broth and kept under constant stirring (150 rpm) for $12 \mathrm{~h}$. Subsequently, $0.1 \mathrm{~mL}$ of cultures from each strain (approximately $10^{8}$ cells) was preincubated for $30 \mathrm{~min}$ with a mixture of $0.5 \mathrm{~mL}$ of $\mathrm{S} 9$ mix or $0.5 \mathrm{~mL}$ of phosphate buffer at $\mathrm{pH} 7.4$ and varying final concentrations of the studied carbamates $(0,0.1,0.2,0.4,0.5$ and $1 \mathrm{mg} / \mathrm{mL}$ ) that had been previously diluted with dimethyl sulfoxide (DMSO). The lowest concentration $(0.1 \mathrm{mg} / \mathrm{mL})$ was selected considering the results of previous assays that demonstrated no toxicity, and the concentration was increased for the remaining assays.

The pre-incubated mix was homogenized into $2 \mathrm{~mL}$ of molten top agar $(0.6 \%$ agar, $0.5 \% \mathrm{NaCl}, 0.5 \mathrm{mM}$ biotin, and $0.05 \mathrm{mM}$ L-histidine) and subsequently distributed homogeneously on the surface of minimal agar plates. The plates 
TABLE 1: Nomenclature, chemical structure and characteristics of ethyl-carbamates.

LQM 919
Chemical structure

were incubated at $37^{\circ} \mathrm{C}$ for $48 \mathrm{~h}$, and the number of reverting colonies in each plate was counted. The criteria for considering carbamates as mutagenic were (1) a dose-dependent increase in the number of reverting colonies or (2) the number of induced reverting colonies greater than or equal to double the amount of reverting colonies found in the negative $(0 \mathrm{mg} / \mathrm{mL})$ control plates $[21,22]$.

Positive controls (known specific mutagens) and negative controls were included in each assay. The specific positive controls were 2 -nitrofluorene $(10 \mu \mathrm{g} /$ plate for TA98 without S9), sodium azide ( $1 \mu \mathrm{g} /$ plate for TA100 and TA1535 without S9), picrolonic acid (100 $\mu$ g/plate for TA1537 without S9), 4Nitroquinoline-N-oxide ( $1 \mu \mathrm{g} /$ plate for TA102 without S9), and 2 -aminoanthracene $(2 \mu \mathrm{g} /$ plate for all strains with S9). All the mutagens were previously diluted in DMSO except for sodium azide, which was dissolved in water. The negative control for all strains was phosphate buffer at $\mathrm{pH} 7.4$ with $2 \%$ DMSO [23].

\section{Results}

3.1. Profiling. Table 2 shows the profiling results of LQM 919 and LQM 996. The qualitative analysis did not show structural alerts that indicated the ability of these ethylcarbamates to either produce damage or bind to biomolecules.

3.2. Mutagenicity Predictions. Table 3 shows the endpoint predictions of mutagenicity in six strains of S. typhimurium. The ethyl-carbamates studied were negative $(p<0.05)$ for the mutagenicity endpoint in all strains studied with and without simulation of metabolic activation using S9.

3.3. Carcinogenicity Predictions. The read across predictions of carcinogenicity in rats were negative for LQM 919 ( $\mathrm{p}<$ 0.05 , moderate confidence) as well as for LQM 996 ( $\mathrm{p}<$ 0.001 , strong confidence). Figure 1 shows the summary carcinogenicity predictions from LQM 919 and neighbours (analogues) used for read-across analysis (a similar result was observed for the LQM 996).

3.4. In vitro Ames' Assay. Tables 4 and 5 show the average number of reverting colonies in strains exposed to different LQM 919 and LQM 996 concentrations with and without S9 metabolic activation, respectively. The number of sponta- neous reverting colonies at all the concentrations used for both carbamates did not show a concentration-dependent increase; moreover, this number was not greater than or equal to double the number of spontaneous reverting colonies of each strain in the respective negative control. In general, greater concentrations of these ethyl-carbamates resulted in thinning out of background growth.

\section{Discussion}

The genotoxicity induced by pesticides is considered one of the most severe undesirable effects in mammals. When a pesticide reacts with nuclear DNA, it is considered potentially mutagenic and carcinogenic to the exposed organisms; therefore, its use in therapeutics may be limited. For the aforementioned reasons, when the use of new pesticides is proposed, their potential as a possible mutagen and carcinogen should be evaluated before being widely used in the environment. In the present study, in silico predictive analyses and studies on in vitro effects showed that the evaluated ethyl-carbamates do not present a mutagenic or carcinogenic risk.

Most mutagens and carcinogens or their metabolites covalently bind to cellular macromolecules such as DNA and proteins before producing changes in their structure, sequence, or function [24]. Some ethyl-carbamates such as urethane reportedly bind to liver cell DNA before producing tumors in mouse liver or to lung cell DNA before producing tumors in rat lungs $[25,26]$. In this study, read-acrossanalysis revealed no DNA-binding or protein-binding alerts in the molecular structure of LQM 919 and LQM 996. These results suggest that these ethyl-carbamates have a low probability of affecting DNA replicative processes that could alter its sequence.

High levels of estrogen have been associated with tumor production both in experimental models and in epidemiological studies in humans [27-29]. One of the mechanisms of estrogen carcinogenicity is its binding to the specific nuclear receptor ER- $\alpha$, exerting a potent stimulus on cell proliferation through increased production of growth factors [30]. Some pesticides such as Acetochlor, Dicofol, Endosulfan, Propoxur, and Lindane mimic estrogen activity in terms of structure and function (xenoestrogens) as these can bind estrogen receptors and stimulate tumor production [31, 32]. In silico analysis did not show structural alerts that indicate 
TABLE 2: Profiling results of Structure-Activity Relationship (SAR) analysis of ixodicidal ethyl-carbamates.

\begin{tabular}{lcc}
\hline Chemical or biological mechanism & LQM 919 & LQM 996 \\
\hline DNA binding by OECD & No alert found & No alert found \\
Estrogen receptor binding & Non binder, without OH or NH2 group & Non binder, without OH or NH2 group \\
Protein binding by OECD & No alert found & No alert found \\
Superfragments & No superfragment & No superfragment \\
Toxic hazard classification by Cramer (original) & High (class III) & High (class III) \\
US-EPA new chemical categories & Neutral organics & Neutral organics \\
\hline
\end{tabular}

TABle 3: Predictions of mutagenicity of ethyl-carbamates on different strains of Salmonella typhimurium with and without S9 metabolic activation.

\begin{tabular}{|c|c|c|c|c|c|}
\hline CARBAMATE & Strain & With S9 activation & $\mathrm{P}$-value & Without $\mathrm{S} 9$ activation & $\mathrm{P}$-value \\
\hline \multirow{6}{*}{ LQM 919} & TA 97 & Negative & $\mathrm{p}<0.001 * * *$ & Negative & $\mathrm{p}=0.100$ \\
\hline & TA 98 & Negative & $\mathrm{p}<0.001 * * *$ & Negative & $\mathrm{p}<0.05 *$ \\
\hline & TA 102 & Negative & $\mathrm{p}<0.01 * *$ & Negative & $\mathrm{p}<0.01 * *$ \\
\hline & TA 1535 & Negative & $\mathrm{p}<0.001 * * *$ & Negative & $\mathrm{p}<0.01 * *$ \\
\hline & TA 1537 & Negative & $\mathrm{p}<0.001 * * *$ & Negative & $\mathrm{p}<0.05 *$ \\
\hline & TA 1538 & Negative & $\mathrm{p}<0.001 * * *$ & Negative & $\mathrm{p}<0.001 * * *$ \\
\hline \multirow{6}{*}{ LQM 996} & TA 97 & Negative & $\mathrm{p}<0.001 * * *$ & Negative & $\mathrm{p}<0.001 * * *$ \\
\hline & TA 98 & Negative & $\mathrm{p}<0.001 * * *$ & Negative & $\mathrm{p}<0.01 * *$ \\
\hline & TA 102 & Negative & $\mathrm{p}<0.01 * *$ & Negative & $\mathrm{p}<0.001 * * *$ \\
\hline & TA 1535 & Negative & $\mathrm{p}<0.001 * * *$ & Negative & $\mathrm{p}<0.01 * *$ \\
\hline & TA 1537 & Negative & $\mathrm{p}<0.001 * * *$ & Negative & $\mathrm{p}<0.01 * *$ \\
\hline & TA 1538 & Negative & $\mathrm{p}<0.01 * *$ & Negative & $\mathrm{p}<0.001 * * *$ \\
\hline
\end{tabular}

$*$ moderate confidence; $* *$ strong confidence; $* * *$ very strong confidence.

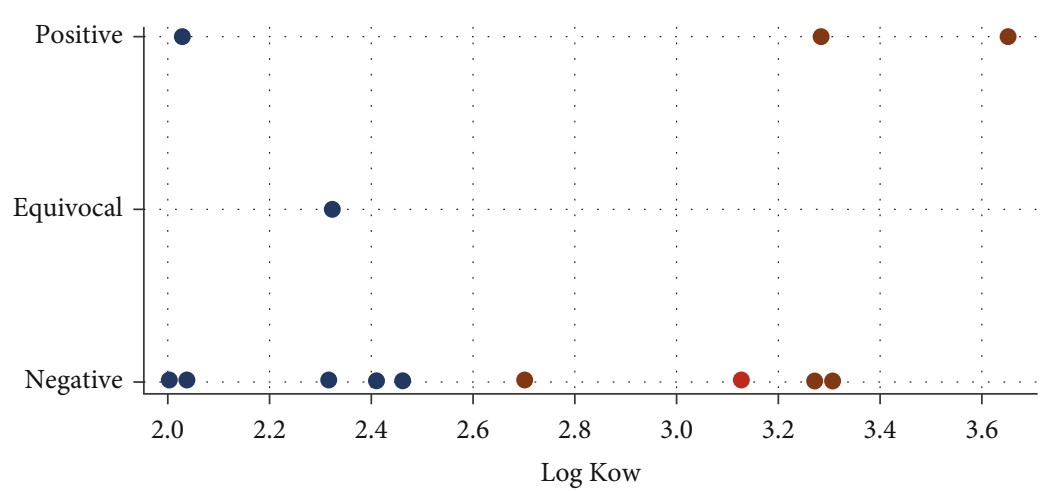

FIGURE 1: Read across prediction of summary carcinogenicity from LQM 919. The red dot represents the predicted value for target chemical (LQM 919), brown dots represent the observed value for the target neighbours (analogues) used for read-across and blue dots represent the experimental results available for the analogues but not used for read-across. Predicted LQM 919 value: "Negative", p $<0.001$.

probable estrogen receptor binding in the ethyl-carbamates studied; therefore, the risk that they can induce cancer by this mechanism is low. In addition to the above, the read across prediction of carcinogenicity in rats was negative for both ethyl-carbamates; therefore, their chemical structure does not seem to present a risk of cancer induction either. However, other alerts indicated possible toxicity. According to the Cramer classification (estimation of the threshold of toxicological concern) these compounds were categorized within class III, which includes compounds with a chemical structure that does not allow an initial impression of safety, and suggests significant toxicity. The US-EPA New Chemical Categories prediction indicated that these are neutral organic compounds related to probable environmental toxicity for some aquatic species [24].

The Ames' assay is used worldwide to determine the mutagenic capability of pesticides $[33,34]$. This test is highly sensitive, and depending on the strain of S. typhimurium 


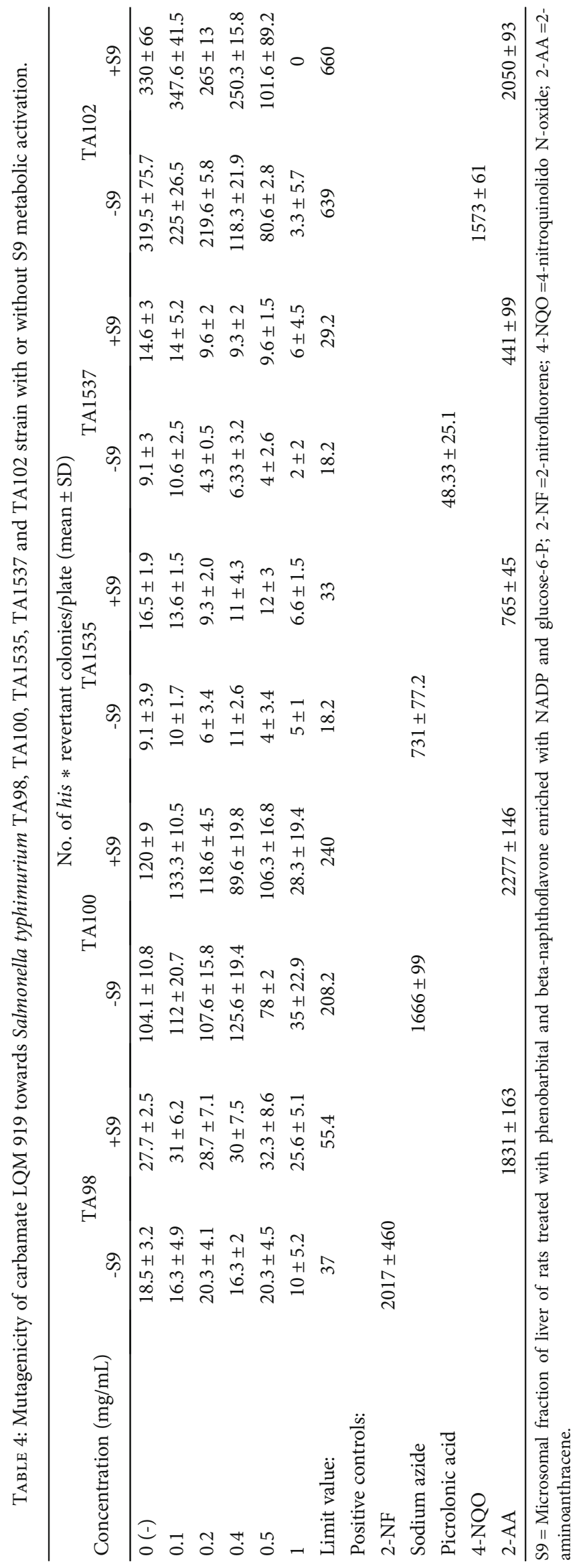




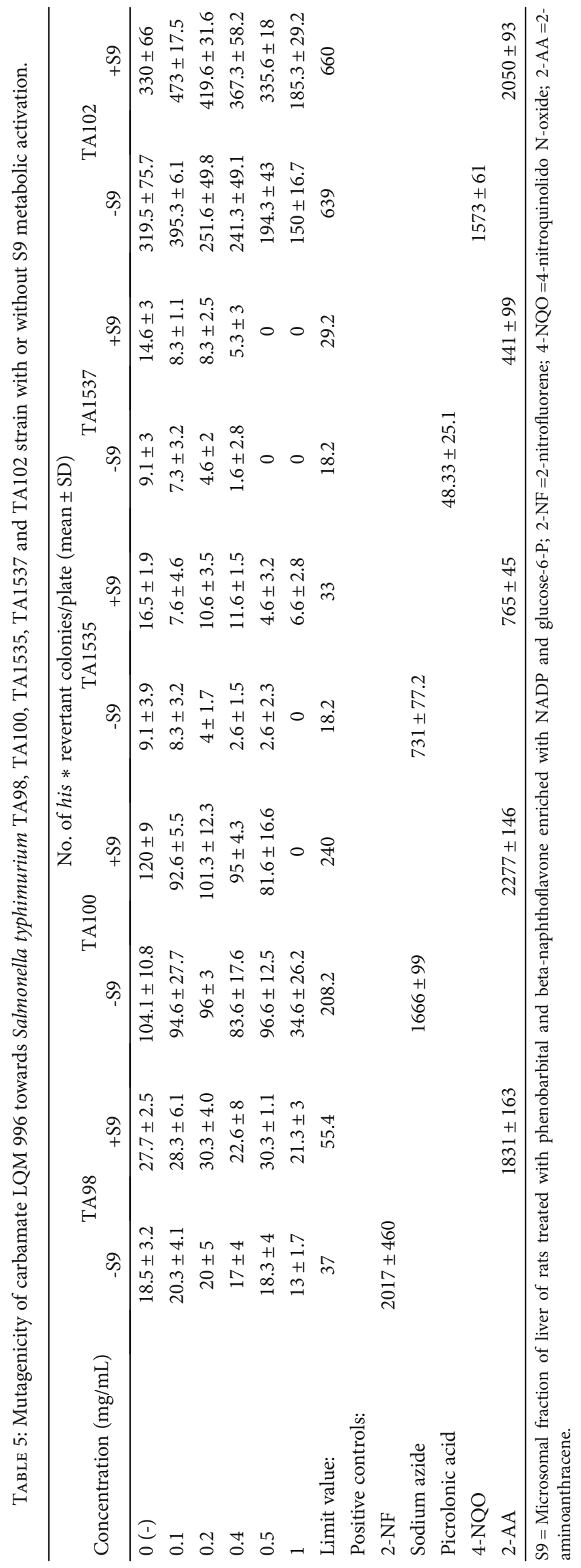


used, it can reveal different types of mutations. Strains TA98, TA1535, and TA1538 are sensitive to frameshift mutations, strains TA97, TA100, and TA1535 are sensitive to C/G base pair exchanges, and strain TA102 is sensitive to A/T base pair exchanges and mutations caused by oxidative damage [22, $35]$. In this study, the results of read-across analysis predicted that the evaluated ethyl-carbamates have no mutagenic potential in any of the six strains of S. typhimurium studied. These predictions were consistent with the results obtained in the Ames in vitro assay, and in both cases, metabolic activation of ethyl-carbamates was considered. Some carbamates such as Propoxur, Carbaryl, and Methomyl acquire mutagenic activity when subjected to exogenous metabolic activation [36, 37]. We did not observe differences in spontaneous reversion induced by the ethyl-carbamates under study with or without exogenous metabolic activation. The in silico and in vitro results obtained in this study indicated the absence of mutagenic activity in the ethyl-carbamates or their metabolites.

In previous studies, we observed that the ethylcarbamates used in this study induce an increase in micronucleated reticulocytes in rats, which could be the result of DNA damage [10]. Furthermore, we observed an increase in thiobarbituric acid reactive species (oxidative stress) that can cause damage to macromolecules including DNA [8]. However, the results of the present study suggest that these ethyl-carbamates did not represent a risk for cancer in mammals.

\section{Conclusions}

In silico studies predicted that the ethyl-carbamates LQM 919 and LQM 996 are negative for mutagenicity and carcinogenicity. The in vitro Ames test confirmed the absence of mutagenic capacity in these compounds.

\section{Data Availability}

The data used to support the findings of this study are available from the corresponding author upon request.

\section{Conflicts of Interest}

The authors declare that there is no conflict of interest regarding the publication of this paper.

\section{Acknowledgments}

We would like to thank Sandra Luz Hernández Ojeda from Instituto de Investigaciones Biomédicas, UNAM and César Cuenca Verde from Facultad de Estudios Superiores Cuautitlán, UNAM for their technical assistance. This study was supported by PAPIIT/UNAM (Projects No. IT201420, IN202020, and IN223420).

\section{References}

[1] A. Amberg, "In silico methods," in Drug Discovery and Evaluation: Safety and Pharmacokinetic Assays, pp. 1273-1296, Springer, 2013.
[2] P. Deplazes, J. Eckert, A. Mathis, G. Samson-Himmelstjerna, and H. von Zahner, Parasitology in Veterinary Medicine, Wageningen Academic Publishers, Wageningen, Netherlands, 2016.

[3] M. A. Taylor, R. L. Coop, and R. L. Wall, Veterinary Parasitology, Wiley Blackwell, Oxford, 4th edition, 2015.

[4] M. G. Prado-Ochoa, M. A. Muñoz-Guzmán, V. H. AbregoReyes et al., "Effect of new ethyl and methyl carbamates on biological parameters and reproduction of the cattle tick Rhipicephalus microplus," Veterinary Parasitology, vol. 194, no. 1, pp. 49-57, 2013.

[5] I. E. Pérez-González, M. G. Prado-Ochoa, M. A. Muñoz-Guzmán et al., "Effect of new ethyl and methyl carbamates on Rhipicephalus microplus larvae and adult ticks resistant to conventional ixodicides," Veterinary Parasitology, vol. 199, no. 3-4, pp. 235-241, 2014.

[6] S. L. Iturbe-Requena, M. G. Prado-Ochoa, A. M. VelázquezSánchez et al., "Oogenesis and embryogenesis inhibition induced by two new ethyl-carbamates in the cattle tick Rhipicephalus microplus," Ticks and Tick-borne Diseases, vol. 11, no. 2, article 101326, 2020.

[7] M. G. Prado-Ochoa, P. Ramírez-Noguera, R. Díaz-Torres et al., "The action of two ethyl carbamates on acetylcholinesterase and reproductive organs of Rhipicephalus microplus," Veterinary Parasitology, vol. 199, no. 3-4, pp. 215-224, 2014.

[8] M. G. Prado-Ochoa, V. H. Abrego-Reyes, A. M. VelázquezSánchez et al., "Subchronic Toxicity Study in Rats of Two New Ethyl-Carbamates with Ixodicidal Activity," BioMed Research International, vol. 2014, 12 pages, 2014.

[9] M. G. Prado-Ochoa, R. A. Gutiérrez-Amézquita, V. H. Abrego-Reyes et al., "Assessment of acute oral and dermal toxicity of 2 ethyl-carbamates with activity againstRhipicephalus microplusin rats," BioMed Research International, vol. 2014, Article ID 956456, 10 pages, 2014.

[10] M. G. Prado-Ochoa, M. A. Muñoz-Guzmán, V. H. VázquezValadez et al., "Genotoxicity and cytotoxicity assessment of new ethyl-carbamates with ixodicidal activity," Mutation Research, Genetic Toxicology and Environmental Mutagenesis, vol. 807, pp. 8-14, 2016.

[11] T. Shen, T. Jiang, M. Long et al., "A curcumin derivative that inhibits vinyl carbamate-induced lung CarcinogenesisviaActivation of the Nrf2 protective response," Antioxidants \& Redox Signaling, vol. 23, no. 8, pp. 651-664, 2015.

[12] M. T. Leithauser, A. Liem, B. C. Stewart, E. C. Miller, and J. A. Miller, "1,N6formation, mutagenicity and murine tumor induction as indicators of the generation of an electrophilic epoxide metabolite of the closely related carcinogens ethyl carbamate (urethane) and vinyl carbamate," Carcinogenesis, vol. 11, no. 3, pp. 463-473, 1990.

[13] E. Angeles, P. Martınez, J. Keller et al., "Ethyl and methylphenylcarbamates as antihelmintic agents: theoretical study for predicting their biological activity by PM3," Journal of Molecular Structure: THEOCHEM, vol. 504, no. 1-3, pp. 141-170, 2000.

[14] S. D. Dimitrov, R. Diderich, T. Sobanski et al., "QSAR toolbox - workflow and major functionalities," $S A R$ and QSAR in Environmental Research, vol. 27, no. 3, pp. 203-219, 2016.

[15] T. W. Schultz, P. Amcoff, E. Berggren et al., "A strategy for structuring and reporting a read-across prediction of toxicity," Regulatory Toxicology and Pharmacology, vol. 72, no. 3, pp. 586-601, 2015.

[16] A. P. Worth, C. J. Van Leeuwen, and T. Hartung, "The prospects for using (Q) SARs in a changing political environment-high 
expectations and a key role for the European Commission's joint research Centre," SAR and QSAR in Environmental Research, vol. 15, no. 5-6, pp. 331-343, 2010.

[17] M. K. Heris and S. Rahnamayan, "Multi-objective Optimal Control of Dynamic Integrated Model of Climate and Economy: Evolution in Action," in 2020 IEEE Congress on Evolutionary Computation (CEC), pp. 1-8, Glasgow, United Kingdom, United Kingdom, 2020.

[18] J. Ye, "Multicriteria decision-making method using the Dice similarity measure based on the reduct intuitionistic fuzzy sets of interval-valued intuitionistic fuzzy sets," Applied Mathematical Modelling, vol. 36, no. 9, pp. 4466-4472, 2012.

[19] D. M. Maron and B. N. Ames, "Revised methods for the Salmonella mutagenicity test," Mutation Research/Environmental Mutagenesis and Related Subjects, vol. 113, no. 3-4, pp. 173215, 1983.

[20] D. Escobar-Garcia, R. Camacho-Carranza, I. Pérez, V. Dorado, M. Arriaga-Alba, and J. J. Espinosa-Aguirre, "S9 induction by the combined treatment with cyclohexanol and albendazole," Mutagenesis, vol. 16, no. 6, pp. 523-528, 2001.

[21] L. D. Claxton, J. Allen, A. Auletta, K. Mortelmans, E. Nestmann, and E. Zeiger, "Guide for the Salmonella typhimurium/mammalian microsome tests for bacterial mutagenicity," Mutation Research/Genetic Toxicology, vol. 189, no. 2, pp. 83-91, 1987.

[22] OECD, "Test No. 471: Bacterial Reverse Mutation Test," in Ninth Addendum to the OECD Guidelines for the Testing of Chemicals, Section 4, OECD Publishing, 2020.

[23] L. R. Williams and J. E. Preston, Interim Procedures for Conducting the Salmonella/Microsomal Mutagenicity Assay (Ames Test), US Environmental Protection Agency, Environmental Monitoring Systems Laboratory, 1983.

[24] R. Diderich, "Tools for category formation and read-across: overview of the OECD (Q) SAR application toolbox," in In Silico Toxicology Principles and Applications, M. T. D. Cronin and J. C. Madden, Eds., pp. 385-405, RSC Publishing, Cambridge, 2010.

[25] L. C. Pan, H. Y. Xiao, W. J. Yin, and Z. Lin, "Correlation between HSD17B4 expression in rat liver cancer tissues and inflammation or proliferation," European Review for Medical and Pharmacological Sciences, vol. 22, pp. 3386-3393, 2018, https://www.europeanreview.org/wp/wp-content/uploads/ 3386-3393-1.pdf.

[26] P. Parashar, C. B. Tripathi, M. Arya et al., "A facile approach for fabricating CD44-targeted delivery of hyaluronic acidfunctionalized PCL nanoparticles in urethane-induced lung cancer: Bcl-2, MMP-9, caspase-9, and BAX as potential markers," Drug Delivery and Translational Research, vol. 9, no. 1, pp. 37-52, 2019.

[27] J. J. Li, S. A. Li, T. D. Oberley, and J. A. Parsons, "Carcinogenic activities of various steroidal and nonsteroidal estrogens in the hamster kidney: relation to hormonal activity and cell proliferation," Cancer Research, vol. 55, pp. 4347-4351, 1995, https:// cancerres.aacrjournals.org/content/55/19/4347.short.

[28] I. H. Russo and J. Russo, "Mammary gland neoplasia in longterm rodent studies," Environmental Health Perspectives, vol. 104, no. 9, pp. 938-967, 1996.

[29] J. D. Yager, "Chapter 3: endogenous estrogens as carcinogens through metabolic activation," JNCI Monographs, vol. 2000, no. 27 , pp. $67-73,2000$.
[30] J. Russo and I. H. Russo, "The role of estrogen in the initiation of breast cancer," The Journal of Steroid Biochemistry and Molecular Biology, vol. 102, no. 1-5, pp. 89-96, 2006.

[31] W. Mnif, A. I. H. Hassine, A. Bouaziz, A. Bartegi, O. Thomas, and B. Roig, "Effect of endocrine disruptor pesticides: a Review," Public Health, vol. 8, no. 6, pp. 2265-2303, 2011.

[32] S. P. Bharathi, H. M. Raj, S. Jain, B. D. Banerjee, T. Ahmed, and V. K. Arora, "Role of pesticides in the induction of tumor angiogenesis," Anticancer Research, vol. 33, p. 231, 2013, http://ar .iiarjournals.org/content/33/1/231.full.pdf+html.

[33] J. Cortés-Eslava, S. Gómez-Arroyo, F. Arenas-Huertero et al., "The role of plant metabolism in the mutagenic and cytotoxic effects of four organophosphorus insecticides in Salmonella typhimurium and in human cell lines," Chemosphere, vol. 92, no. 9, pp. 1117-1125, 2013.

[34] D. Akyl and M. Konuk, "Detection of genotoxicity and mutagenicity of chlorthiophos using micronucleus, chromosome aberration, sister chromatid exchange, and Ames tests," Environmental Toxicology, vol. 30, no. 8, pp. 937-945, 2015.

[35] D. E. Levin, E. Yamasaki, and B. N. Ames, "A new Salmonella tester strain, TA97, for the detection of frameshift mutagens: a run of cytosines as a mutational hot-spot," Mutation Research/Fundamental and Molecular Mechanisms of Mutagenesis, vol. 94, no. 2, pp. 315-330, 1982.

[36] R. D. Blevins, M. Lee, and J. D. Regan, "Mutagenicity screening of five methyl carbamate insecticides and their nitroso derivatives using mutants of Salmonella typhimurium LT2," Mutation Research/Fundamental and Molecular Mechanisms of Mutagenesis, vol. 56, no. 1, pp. 1-6, 1977.

[37] T. C. Wang, C. M. Chiou, and Y. L. Chang, "Genetic toxicity of $\mathrm{N}$-methylcarbamate insecticides and their N-nitroso derivatives," Mutagenesis, vol. 13, no. 4, pp. 405-408, 1998. 